\title{
The crucial role of primary care providers in the long-term follow-up of adult survivors of childhood cancer
}

This article was published in the following Dove Press journal: Cancer Management and Research

\author{
Justin Jain (1D ${ }^{1, *}$ \\ Bessi Qorri iD ${ }^{2, *}$ \\ Myron R Szewczuk (iD) ${ }^{2}$ \\ 'Postgraduate Medical Education, \\ Graduate Diploma and Professional \\ Master in Medical Sciences, School of \\ Medicine, Queen's University, Kingston, \\ ON, Canada; ${ }^{2}$ Department of Biomedical \\ and Molecular Sciences, Queen's \\ University, Kingston, ON, Canada \\ *These authors contributed equally to \\ this work
}

Purpose: The potential physical or psychosocial factors that play a role in the progression of childhood cancer survivors into adulthood are essential in the identification of an effective patient-centred approach to therapy. Despite the presence of guidelines published by the Children's Oncology Group, knowledge of the long-term health sequelae for the care of survivors is sub-optimal. Here, the pertinent clinical issues that may affect survivors of childhood cancer are outlined for primary care providers (PCPs).

Methods: This literature search identified articles using PubMed, EMBASE Ovid, and the Cochrane Library to determine high-quality, multicenter randomized controlled trials, systematic reviews, meta-analyses, and practice guidelines from December 1998 to December 2018. The keywords of the search were primary care providers; childhood cancer survivors; long-term care and mental health. Guidelines and research using retrospective studies are used to compile evidence to address PCP's involvement and to describe the factors involved in the adult onset of psychological disorders in survivors of childhood cancer. A focus of this article is to use the literature that evaluated pediatric cancer survivors for at least five years post diagnosis and had received cancer treatment including chemotherapy, radiation, bone marrow transplant, or surgery. Additional research focused on primary care physicians addressing the care of childhood cancer survivors. Here, we aim to provide PCPs and physicians with a critical yet concise update on the recent advancements for this important healthcare topic. This paper presents an overview of previously published reviews and, as such, requires no ethics approval.

Results: Childhood cancer survivors can develop symptoms of depression and suffer from low self-esteem from their diagnosis and treatment regimens. These symptoms can result in functional impairment. Child diagnosis also affects parental health, resulting in the experience of psychological, emotional and traumatic stress. The feeling of helplessness and guilt on parents leads to the potentiation of depression on the child survivor.

Conclusions: Primary care providers, in collaboration with clinician specialists, must be vigilant in providing consistent long-term care. This approach will ensure clear constant communication to help address the challenges faced by the families and survivors as they progress through adulthood.

Implications for cancer survivors: Encouraging primary care providers to become knowledgeable and comfortable in utilizing appropriate resources is achieved through consultation with oncology or psychiatric specialists or with online resources for safer management of childhood cancer survivors. The implications for this patient population would ultimately allow for a more patient-centred approach to therapy.

Keywords: primary care providers, childhood cancer survivors, long-term care, mental health 


\section{Introduction}

Childhood cancer survivors (CCS) can face many psychological and social issues well into adulthood and represent a particularly vulnerable patient population. ${ }^{1}$ Without proper and continuous support, this subset of patients is at an increased risk of developing post-traumatic stress disorder (PTSD) including anxiety and depression. ${ }^{2}$ A recent review of the adverse mental health effects of Canadian CCS indicated that this patient population had significantly higher rates of mental health visits to family medicine physicians and psychiatrists compared to the general population. ${ }^{3}$ Fortunately, childhood cancer patient outcomes have substantially improved, with childhood cancer mortality significantly decreasing over the last few decades. These improvements are owed in part to advancements in detection and treatment options, mainly due to the increased availability of chemotherapy, radiation and bone marrow transplants. ${ }^{4-6}$ Central to this success is the crucial role of primary care providers (PCPs) in providing appropriate screening and the most effective treatment options, catering not only to the cancer survivors but also to the family. ${ }^{7,8}$ However, a significant gap in our understanding remains and relates to the psychological challenges faced by survivors. More specifically, these challenges include adapting to the biological, social, and environmental changes that are a result of their cancer diagnosis and subsequent treatment.

Here, we aim to surmise the psychological effects faced by childhood cancer survivors, particularly as they transition into adolescence and adulthood. By doing so, we hope to enhance PCP knowledge on this subset of patients and the multitude of risk factors that can potentiate into clinical psychiatric disorders. We encourage PCPs to continue collaborating with specialist physicians to attain a thorough patient background that will ultimately enhance patient care through early intervention, screening and developing a long-term care plan through a patientcentered approach that is unique to each patient.

\section{Understanding the unique patient population}

Childhood cancer survivors represent a unique patient population that is subject to several long-term adverse effects that negatively impact their overall quality of life (QOL). Cancer treatment regimens themselves are intensive and can interfere with a patient's daily routine, impact their education, social interactions and overall physical and mental health. ${ }^{9}$ Collectively these interferences can stunt otherwise healthy childhood development and can potentially manifest in later years as psychological disorders or other complications. As such, we have outlined the main aspects of healthy childhood development that can be affected and can impede normal physical and psychological growth.

\section{Education}

Depending on the time of cancer diagnosis and initiation and nature of the treatment regimen, many childhood cancer patients start school several years later than their peers or miss at least two terms of school. ${ }^{9}$ Absence from school as a result of treatment has been correlated with lower grades following the patient's return to school when compared to their healthy peers. ${ }^{9,10}$ A retrospective multicenter Canadian study found that CCS under the age of 17 were more likely to repeat their school grade year and attend learning disability or special education programs. ${ }^{10}$ Adolescents with cancer may demonstrate problems in their self-esteem also associated with this lower schooling. ${ }^{11}$ Children diagnosed with CNS tumours, leukemia, and neuroblastomas were found to require particularly close monitoring as they were associated with the worst outcomes due to neurocognitive deficits from either the cancer itself or the intense treatment including surgery, chemotherapy, or radiation. ${ }^{10,12}$ Not only can this affect the patient's ability to achieve academic success, but it can impact their contribution to society if they are unable to pursue a post-secondary degree or career that requires a strong academic record.

\section{Social effects}

In addition to the hindrance to education, absence from school also results in missing opportunities for social development with peers; this ultimately has long-term effects on an individual's ability to interact with their peers in social settings throughout life. Survivors of acute lymphoblastic leukemia (ALL) treated with chemotherapy were found to be more likely to demonstrate headstrong behavior, inattention-hyperactivity, social withdrawal, and had higher rates of learning difficulties when compared to their siblings. ${ }^{13}$ Male and female CCS have shown differences in the social effects faced, with females reaching psychosexual milestones later than their male counterparts. Male survivors were also found to live with their parents for more extended periods of time; yet, no significant differences were demonstrated in female survivors. However, both sexes 
displayed a delay in first-time marriage and the birth of a first child with $14.5 \%$ of the study cohort reporting to have infertility. ${ }^{14}$ As such, the effects experienced by CCS concerning social interactions can negatively impact survivors from interacting with and forming meaningful relationships with other individuals.

\section{Physical and psychological health effects}

Substantial evidence exists that correlates CCS with an increased risk of adverse physical effects including developing chronic health conditions, such as secondary cancers, cardiovascular disease, renal dysfunction, severe musculoskeletal problems, and endocrinopathies. ${ }^{15}$ It is well known that many cancer treatments including chemotherapy and radiation are toxic to the normal tissues of the body in addition to cancer itself. ${ }^{16}$ The total disease burden of adverse health outcomes of childhood cancer and subsequent oncological follow-ups was examined for both clinical and subclinical disorders. Up to $75 \%$ of CCS had at least one adverse event, and $25 \%$ had five or more adverse events. ${ }^{17}$ Although there is substantial documentation on the elevated risk of physical adverse effects in CCS, there remains to be a significant gap in knowledge regarding the risk of developing mental health problems in this patient population. ${ }^{18}$

There is substantial evidence that shows that there is an increased risk of psychological distress with worsening physical health over time as a result of cancer treatment. ${ }^{19}$ To gain insight into the psychological effects faced by this subset of patients, a pediatric oncologist, internist and psychologist followed up with $130 \mathrm{CCS}$ and reported that $56.2 \%$ of patients had one psychiatric disorder since cancer diagnosis and $35.4 \%$ reported current onset of at least one psychiatric disorder. ${ }^{20}$ The psychiatric disorders reported included anxiety disorders, mood disorders and psychotic episodes which could occur at any time following diagnosis or cancer treatment. ${ }^{20}$ In particular, pediatric cancer survivors of leukemia, brain tumours, bone tumours, sarcoma, Wilms tumour and lymphomas were found at maximum risk of developing psychological issues including depression, somatization, and impaired physical health. ${ }^{21}$ There has also been a striking correlation between CNS cancer survivors and the prevalence of depression and anxiety, antisocial behavior, and regression in social competence. ${ }^{22}$

Patients that develop cardiovascular, endocrine, and pulmonary conditions as a result of radiotherapy and anthracycline exposure or cranial radiotherapy (CRT) on pituitary and endocrine glands also demonstrate an elevated risk of developing depression, anxiety, and posttraumatic stress symptoms (PTSS). ${ }^{2}$ Cancer survivors have also been found to be more likely to use multiple antidepressants than their healthy peers, with survivors diagnosed in adolescence is more likely to use antidepressants than early childhood survivors. ${ }^{23}$

Up to $60 \%$ of cancer patients were found to be suffering from at least one serious medically toxic effect of treatment, most commonly involuntary infertility, however, survivors of CNS tumours, bone tumours, and sarcomas are more likely to report functional impairment due to the types of treatments they receive compared to other cancer survivors. ${ }^{16,24} 50 \%$ of CCS who had CNS malignancies had a significant cognitive impairment as per the Childhood Cancer Survivors Study Neurocognitive Questionnaire form (CCSSNCQ), highlighting the need for cognitive rehabilitation. ${ }^{25}$ Survivors of leukemia, CNS tumours, and neuroblastomas also demonstrated higher rates of depression/anxiety and antisocial behaviours. Also, they have diminished social competence following cranial radiation and intrathecal methotrexate. ${ }^{22}$ The insurmountable evidence available that reports a correlation between adverse psychological and physical effects faced by childhood cancer survivors strongly suggests the urgent need for stringent monitoring of psychological well-being of this patient population and to recognize and provide treatment accordingly.

\section{A conceptual model for the development of psychological disorders in adult survivors of childhood cancer}

Studying the risk factors that predispose cancer survivors to depression has resulted in the development of an innovative conceptual, theoretical framework for factors that play a role in CCS development of psychological disorders. This conceptual framework consists of five variables biological, individual, family, community, and global levels of influence with each variable having a different impact on each CCS. ${ }^{26}$ Ultimately this framework, depicted in Figure 1 , assists in determining the risk of developing adulthood depression. Having a framework such as this can assist PCPs in adopting anticipatory risk-based care with proactive prevention for cancer survivors through proper surveillance and scanning for possible reoccurrences of cancer within this patient population. ${ }^{15}$ 


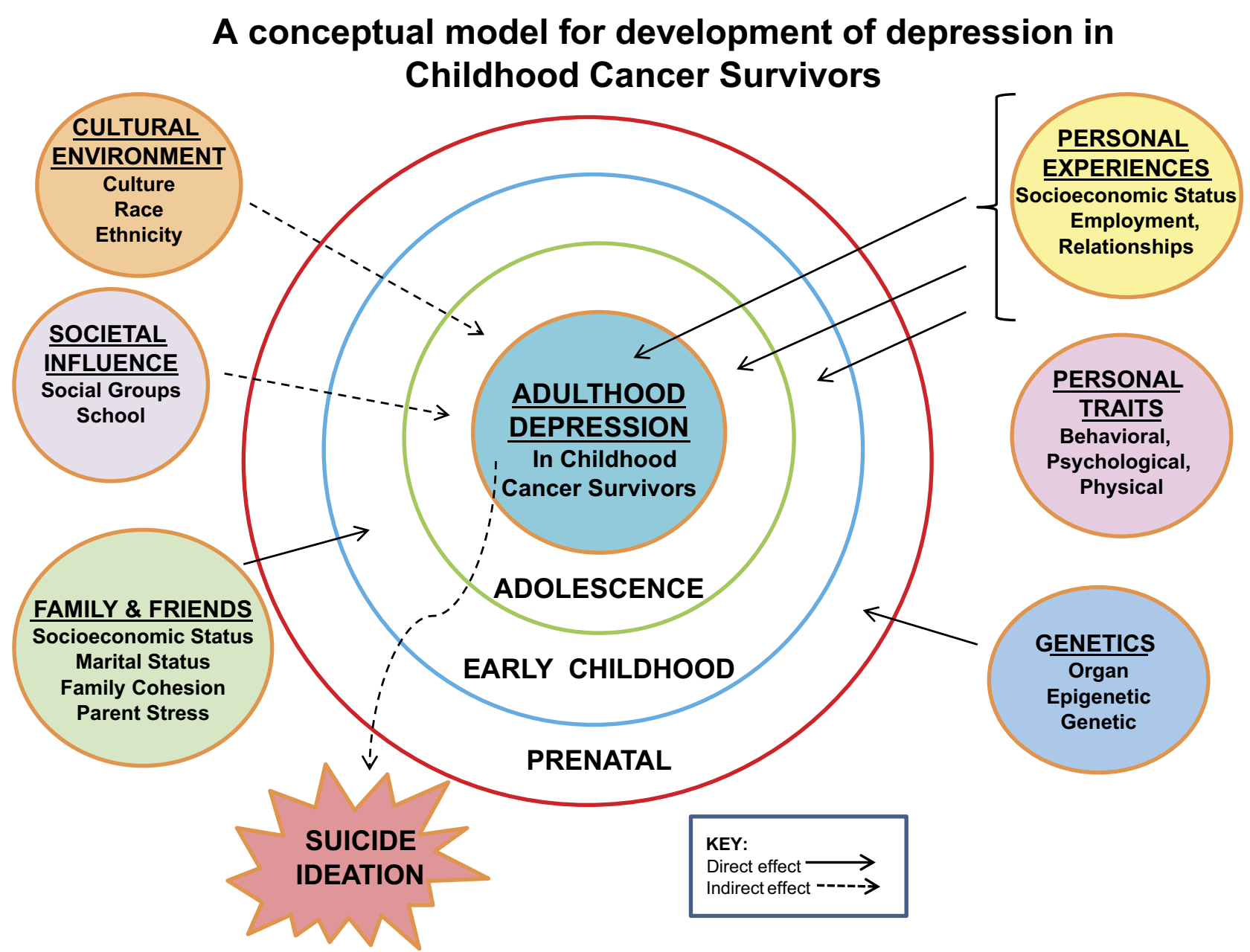

Figure I A conceptual model for the development of depression in childhood cancer survivors (CCS). Building on the framework developed by Kaye EC, Brinkman TM, Baker $\mathrm{JN}^{26}$ we provide a simplified conceptual model for the development of depression in childhood cancer survivors. This proactive model can be used for diagnosing and providing care before the potential onset of depression in CCS. This conceptual model focuses on six variables that can trigger the onset of depression in CCS from the prenatal period to adulthood.

\section{Influence of family}

A child diagnosed with a life-threatening condition such as cancer is faced with many physical and psychological adverse effects. However, this psychological trauma is not exclusive to the patient, as it extends to the family as well. ${ }^{27}$ With children diagnosed with cancer, the parents face a traumatizing experience that can trigger several psychological and physiological processes that ultimately increase the risk of anxiety, PTSD, and depression. ${ }^{28}$ The number and severity of these symptoms were found to increase when parents experienced negative emotions such as self-blame when coping with their child's newly diagnosed illness. ${ }^{28}$ As common as self-blame is in the parent's attempt to cope with a child's diagnosis of cancer, it is also common for parents to express signs and symptoms of depression, with persistent feelings of sadness, anxiety, emptiness, hopelessness, helplessness, and guilt. ${ }^{29}$
Recently, the prevalence of parental depression associated with the diagnosis of a child's cancer has pushed researchers to investigate the long-term implications of parental depression on children diagnosed with cancer. It has been shown that parental distress in the form of anxiety, depression and traumatic stress within the first year following a child's diagnosis is correlated with higher child distress, internalizing and externalizing symptoms, PTSS, and social competence problems up to seven years following diagnosis. ${ }^{30}$ The interesting correlation between childhood cancer survivor outcomes and parental health has been eloquently summarized in Figure 2. Due to this correlation, several screening tools have been developed to help with the identification of families facing psychosocial stress regarding the new diagnosis of their child with cancer. ${ }^{31}$ The Psychosocial Assessment Tool 2.0 (PAT2.0) has been 
reported to be a robust evaluative tool that facilitates a proper measure for familial psychosocial risk among pediatric cancer survivors. ${ }^{32}$ Parents with PTSD can be screened with the PCL-C (PTSD Check List for Civilians), which has been shown to have diagnostic utility. ${ }^{33}$

\section{Suicidal ideation in adult survivors of pediatric cancer}

The conceptual framework for the development of depression in survivors of childhood cancer can be expanded to include the after-effects of depression once a diagnosis has been made, particularly in cases where suicidal ideation (SI) begins. When dealing with mood disorders, an important aspect to consider is the assessment of suicidal risk. In multiple studies, a link between cancer survivors and an increased risk of suicide has been reported when compared with suicides in the general population. ${ }^{34} \mathrm{Up}$ to $8 \%$ of cancer survivors reported suicidal ideation compared to $4.6 \%$ of people in a non-cancer control group population, with a strong correlation between primary CNS cancer and depression. ${ }^{35}$ When evaluating medical records of brain tumour survivors, $11.7 \%$ of survivors reported SI during one of the clinic visits, with some patients screened for SI reporting SI occurrences more than once. ${ }^{36}$ In this study, the average age of SI was 16.9 years, with a significant correlation between patients with a follow-up at an older age and SI.

\section{Primary care physicians: a crucial role in the care of adult survivors of pediatric cancer}

It is evident that pediatric cancer survivors require a multidimensional system of care that focuses on longterm care and follow-up as they progress through life. Currently, there are shared models of care, in which collaboration between pediatric oncologists and PCPs has shown that patients preferred a shared care model for longterm follow-up care of adult survivors of childhood cancer. Thus, it is crucial that improvements in communication between primary care physicians and oncological teams

\section{Psychological and Social factors influencing Pediatric Cancer Survivor and Parental Interrelationship}

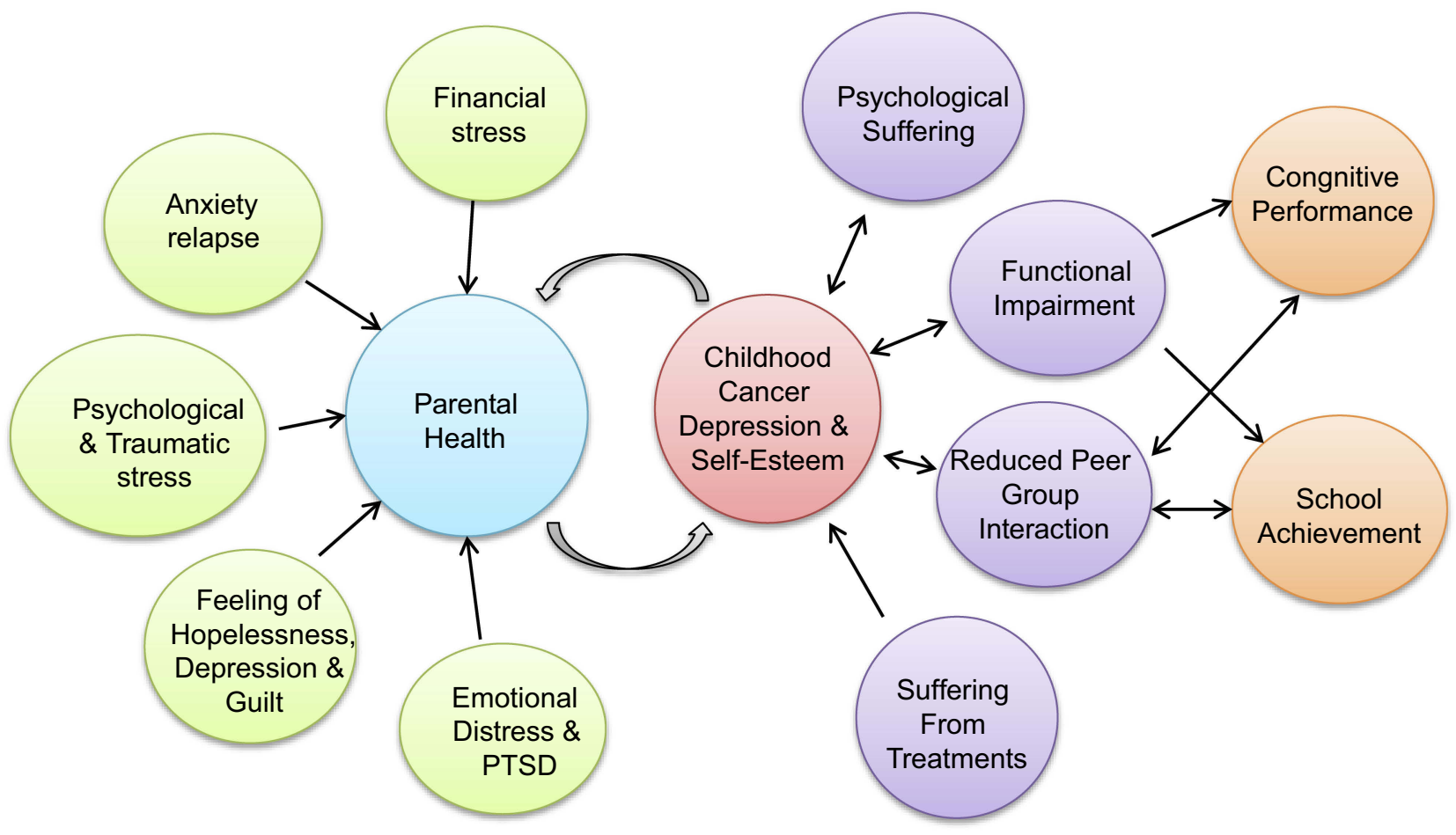

Figure 2 The psychological and social factors influencing the interrelationship between childhood cancer survivors and parents. Childhood cancer survivors can develop symptoms of depression and suffer from low self-esteem from their diagnosis and treatment regimens which can result in functional impairment. Child diagnosis in-turn affects parental health, resulting in experiencing psychological, emotional and traumatic stress - the feeling of helplessness and guilt on parent's leads to potentiation of depression on the child survivor. 
continue so that communication information regarding the diagnosis, treatment, and future screening recommendations for patients reaches the necessary health professionals most quickly and efficiently. ${ }^{37}$ General practitioners have shown a willingness to care for this patient population, with several studies reporting that the majority of physicians (75-97\%) are in favour of participating in a shared care program in collaboration with the pediatric oncology department for follow-ups for adult survivors. ${ }^{38,39}$

However, these collaborative programs can be challenging, particularly when it comes to acquiring patient history. PCPs and treating oncologists have reported difficulties in acquiring an adequate cancer history from the patient. This approach represents a communication barrier between physicians and patients that ultimately leads to inadequate cancer treatment summaries for PCPs to use for their patient treatment. ${ }^{40}$ In North America, an additional challenge exists in which there is a delay between cancer diagnosis and the first cancer-related visit. The likelihood of a patient having a cancer-related visit, or having a general physical examination significantly decreased as the time interval from the original diagnosis increased. ${ }^{41}$

This patient population is particularly vulnerable as they transition from pediatrics to adult care, there is an increased likelihood of loss of continuity in care with providers. ${ }^{42}$ Patients have shown to have increased health care selfefficiency (confidence in health care) when following up with their regular oncologist as a source of care rather than a PCP. Pediatric generalists and PCPs acknowledge that they have a limited mastery of cancer survivorship issues. However, this has left patients with less self-confidence in their health care, feeling less connected to their physician, and as though they have inadequate support. ${ }^{43}$ Preliminary research has shown that cancer survivors are in favour of programs that involve improved coordination between PCPs, oncologists, and patients as they transition from pediatric to adult-based care. ${ }^{44}$ In order to improve the relationship between patients and PCPs, programs should be focused on improving communication and increasing PCP involvement during the treatment of cancer/early survivorship which can help to overcome the low patient confidence in PCPs. ${ }^{45}$

It is recommended that patients have a general clinical follow-up by a PCP every one to two years once they are declared cancer free for up to five years. ${ }^{6}$ This constant follow-up will assist in ensuring continuity of care. The American Family Physician has put out recommendations to help family physicians aid in the surveillance for secondary cancers. Additional areas of focus are to identify late adverse effects from therapy and attend to the psychosocial needs of patients. For this reason, it is fundamental to apply ad hoc surveys ${ }^{46}$ to understand their satisfaction/ dissatisfaction with health professionals. It is essential that care is tailored to survivors based on risks of associated late adverse effects. When considering care for childhood cancer survivors, it is essential that future physician training and education include increasing incorporation of childhood cancer survivors as a patient population during residency physician training programs. ${ }^{40}$ The transition to adult care should be a deliberate systemic process with the creation of a complete summary of the cancer treatment. ${ }^{47}$

\section{Conclusion}

Childhood cancer survivors are at maximum risk of developing anxiety and depression as they progress into adulthood. They are more likely to be self-withdrawn, do poorly in school and develop antisocial behavior, while some may even show suicide ideation. The traumatic experience of cancer diagnosis at an early age can also affect parental health as they experience uncertainty and feel depressed. When pediatric survivors are declared cancer-free, the onus of long-term care falls upon the primary care physician to provide the management of care for not only the survivor patient but also the parents. However, more research is necessary to address issues such as allocation of time, increased exposure to cancer survivors during training, knowledge and increased exposure of cancer survivor guidelines that will help PCPs provide care to this unique patient population.

It is essential that PCPs have increased confidence in the various psychological issues that can affect the CCS and their families, which can be obtained by better understanding the patient population. Encouraging primary care physicians to become knowledgeable and comfortable in utilizing appropriate resources through consultation with oncological or psychiatric specialists or online resources can allow for safer management of this patient population. Ultimately, this would allow for a more patient-centred approach to therapy. As a result, primary care physicians, in collaboration with specialists can be vigilant in providing consistent long-term care by ensuring there is clear constant written or oral communication to help address the challenges faced by the families and survivors as they progress through adulthood. 


\section{Abbreviation list}

ALL, Acute lymphoblastic leukemia; CCS, Childhood cancer survivor; CCSS-NCQ, Childhood Cancer Survivors Study Neurocognitive Questionnaire form; CNS, Central nervous system; CRT, Cranial radiotherapy; PAT2.0, Psychosocial Assessment Tool 2.0; PCL-C, PTSD Checklist for Civilians; PCP, Primary care provider; PTSD, Post-traumatic stress disorder; PTSS, Post-traumatic stress symptoms; QOL, Quality of life; SI, Suicide ideation.

\section{Compliance with ethical standards}

This article does not contain any studies with human participants or animals performed by any of the authors.

\section{Acknowledgments}

This work was supported in part by grants to MR Szewczuk from the Natural Sciences and Engineering Research Council of Canada (NSERC), a private sector cancer funding from the Josefowitz Family and Encyt Technologies, Inc. B Qorri is a recipient of the Queen's Graduate Award (QGA), the 2017 Terry Fox Research Institute Transdisciplinary Training Program in Cancer Research and the 2018 Dean's Doctoral Award. Justin Jain and Bessi Qorri are contributing first authors on this paper.

\section{Author contributions}

All authors contributed toward data analysis, drafting and revising the paper, gave final approval of the version to be published, and agree to be accountable for all aspects of the work.

\section{Disclosure}

The authors report no conflicts of interest in this work.

\section{References}

1. Zebrack BJ. Psychological, social, and behavioral issues for young adults with cancer. Cancer. 2011;117(S10):2289-2294. doi:10.1002 cncr. 26056

2. Vuotto SC, Krull KR, Li C, et al. Impact of chronic disease on emotional distress in adult survivors of childhood cancer: a report from the Childhood Cancer Survivor Study. Cancer. 2017;123 (3):521-528. doi:10.1002/cncr.30348

3. Nathan PC, Nachman A, Sutradhar R, et al. Adverse mental health outcomes in a population-based cohort of survivors of childhood cancer. Cancer. 2018. doi:10.1002/cncr.31279

4. Eiser C. Practitioner review: long-term consequences of childhood cancer. J Child Psychol Psychiatry. 1998;39(5):621-633.

5. Siegel RL, Miller KD, Jemal A. Cancer statistics, 2018. CA Cancer J Clin. 2018;68(1):7-30. doi:10.3322/caac.21442

6. Wallace WHB, Blacklay A, Eiser C, et al. Developing strategies for long term follow up of survivors of childhood cancer. BMJ. 2001;323 (7307):271-274.
7. Kopp LM, Gupta P, Pelayo-Katsanis L, Wittman B, Katsanis E. Late effects in adult survivors of pediatric cancer: a guide for the primary care physician. Am J Med. 2012;125(7):636-641. doi:10.1016/j. amjmed.2012.01.013

8. Svedberg P, Einberg E-L, Wärnestål P, et al. Support from healthcare services during transition to adulthood-experiences of young adult survivors of pediatric cancer. Eur J Oncol Nurs. 2016;21:105-112. doi:10.1016/j.ejon.2016.02.008

9. Yilmaz MC, Sari HY, Cetingul N, Kantar M, Erermis S, Aksoylar S Determination of school-related problems in children treated for cancer J Sch Nurs. 2014;30(5):376-384. doi:10.1177/1059840513506942

10. Barrera M, Shaw AK, Speechley KN, Maunsell E, Pogany L. Educational and social late effects of childhood cancer and related clinical, personal, and familial characteristics. Cancer. 2005;104 (8):1751-1760. doi:10.1002/cncr.21390

11. Tremolada M, Taverna L, Bonichini S, Basso G, Pillon M. Selfesteem and academic difficulties in preadolescents and adolescents healed from paediatric leukaemia. Cancers. 2017;9(6):55. doi:10.3390/cancers9060055

12. Mulhern RK, Merchant TE, Gajjar A, Reddick WE, Kun LE. Late neurocognitive sequelae in survivors of brain tumours in childhood. Lancet Oncol. 2004;5(7):399-408. doi:10.1016/ S1470-2045(04)01507-4

13. Jacola LM, Edelstein K, Liu W, et al. Cognitive, behaviour, and academic functioning in adolescent and young adult survivors of childhood acute lymphoblastic leukaemia: a report from the Childhood Cancer Survivor Study. The Lancet Psychiatry. 2016;3 (10):965-972. doi:10.1016/S2215-0366(16)30283-8

14. Dieluweit U, Debatin KM, Grabow D, et al. Social outcomes of longterm survivors of adolescent cancer. Psycho-Oncology. 2010;19 (12):1277-1284. doi:10.1002/pon.1692

15. Oeffinger KC, Mertens AC, Sklar CA, et al. Chronic health conditions in adult survivors of childhood cancer. $N$ Engl J Med. 2006;355 (15):1572-1582. doi:10.1056/NEJMsa060185

16. Crom DB, Chathaway DK, Tolley EA, Mulhern RK, Hudson MM. Health status and health-related quality of life in long-term adult survivors of pediatric solid tumors. Int J Cancer. 1999;83(S12):25-31.

17. Geenen MM, Cardous-Ubbink MC, Kremer LC, et al. Medical assessment of adverse health outcomes in long-term survivors of childhood cancer. JAMA. 2007;297(24):2705-2715. doi:10.1001/ jama.297.24.2705

18. Park EM, Rosenstein DL. Depression in adolescents and young adults with cancer. Dialogues Clin Neurosci. 2015;17(2):171.

19. Brinkman T, Zhu L, Zeltzer L, et al. Longitudinal patterns of psychological distress in adult survivors of childhood cancer. $\mathrm{Br}$ $J$ Cancer. 2013;109(5):1373. doi:10.1038/bjc.2013.623

20. Bagur J, Massoubre C, Casagranda L, Faure-Conter C, TrombertPaviot B, Berger C. Psychiatric disorders in 130 survivors of childhood cancer: preliminary results of a semi-standardized interview. Pediatr Blood Cancer. 2015;62(5):847-853. doi:10.1002/pbc.25425

21. Zeltzer LK, Recklitis C, Buchbinder D, et al. Psychological status in childhood cancer survivors: a report from the Childhood Cancer Survivor Study. J Clin Oncol. 2009;27(14):2396. doi:10.1200/ JCO.2008.21.1433

22. Schultz KAP, Ness KK, Whitton J, et al. Behavioral and social outcomes in adolescent survivors of childhood cancer: a report from the childhood cancer survivor study. J Clin Oncol. 2007;25 (24):3649-3656. doi:10.1200/JCO.2006.09.2486

23. Deyell RJ, Lorenzi M, Ma S, et al. Antidepressant use among survivors of childhood, adolescent and young adult cancer: a report of the Childhood, Adolescent and Young Adult Cancer Survivor (CAYACS) research program. Pediatr Blood Cancer. 2013;60(5):816-822. doi:10.1002/pbc.24446

24. Hudson MM, Mertens AC, Yasui Y, et al. Health status of adult long-term survivors of childhood cancer: a report from the Childhood Cancer Survivor Study. JAMA. 2003;290(12):1583-1592. doi:10.1001/jama.290.12.1583 
25. Ellenberg L, Liu Q, Gioia G, et al. Neurocognitive status in long-term survivors of childhood CNS malignancies: a report from the Childhood Cancer Survivor Study. Neuropsychology. 2009;23 (6):705. doi:10.1037/a0016674

26. Kaye EC, Brinkman TM, Baker JN. Development of depression in survivors of childhood and adolescent cancer: a multi-level life course conceptual framework. Support Care Cancer. 2017;25 (6):2009-2017. doi:10.1007/s00520-017-3659-y

27. Long KA, Marsland AL. Family adjustment to childhood cancer: a systematic review. Clin Child Fam Psychol Rev. 2011;14 (1):57-88. doi:10.1007/s10567-010-0082-z

28. Greening L, Stoppelbein L. Brief report: pediatric cancer, parental coping style, and risk for depressive, posttraumatic stress, and anxiety symptoms. J Pediatr Psychol. 2007;32(10):1272-1277. doi:10.1093/ jpepsy/jsm057

29. Jantien Vrijmoet-Wiersma C, van Klink JM, Kolk AM, Koopman HM, Ball LM, Maarten Egeler R. Assessment of parental psychological stress in pediatric cancer: a review. J Pediatr Psychol. 2008;33(7):694-706. doi:10.1093/jpepsy/jsn007

30. Sultan S, Leclair T, Rondeau E, Burns W, Abate C. A systematic review on factors and consequences of parental distress as related to childhood cancer. Eur J Cancer Care (Engl). 2016;25(4):616-637. doi:10.1111/ecc.12361

31. Pierce L, Hocking MC, Schwartz LA, Alderfer MA, Kazak AE, Barakat LP. Caregiver distress and patient health-related quality of life: psychosocial screening during pediatric cancer treatment. Psycho-Oncology. 2017;26(10):1555-1561. doi:10.1002/pon.4171

32. Gilleland J, Reed-Knight B, Brand S, et al. Assessment of family psychosocial functioning in survivors of pediatric cancer using the PAT2. 0. Psycho-Oncology. 2013;22(9):2133-2139. doi:10.1002/pon.3265

33. Manne SL, Du Hamel K, Gallelli K, Sorgen K, Redd WH. Posttraumatic stress disorder among mothers of pediatric cancer survivors: diagnosis, comorbidity, and utility of the PTSD checklist as a screening instrument. J Pediatr Psychol. 1998;23(6):357-366.

34. Shah SS, Dellarole A, Peterson EC, et al. Long-term psychiatric outcomes in pediatric brain tumor survivors. Child's Nerv Syst. 2015;31(5):653-663. doi:10.1007/s00381-015-2669-7

35. Recklitis CJ, Diller LR, Li X, Najita J, Robison LL, Zeltzer L. Suicide ideation in adult survivors of childhood cancer: a report from the childhood cancer survivor study. J Clin Oncol. 2010;28 (4):655. doi:10.1200/JCO.2009.22.8635

36. Brinkman TM, Liptak CC, Delaney BL, Chordas CA, Muriel AC, Manley PE. Suicide ideation in pediatric and adult survivors of childhood brain tumors. $J$ Neurooncol. 2013;113(3):425-432. doi:10.1007/s11060-013-1130-6
37. Blaauwbroek R, Tuinier W, Meyboom-de Jong B, Kamps WA, Postma A. Shared care by paediatric oncologists and family doctors for long-term follow-up of adult childhood cancer survivors: a pilot study. Lancet Oncol. 2008;9(3):232-238. doi:10.1016/S1470-2045(08)70034-2

38. Blaauwbroek R, Zwart N, Bouma M, Meyboom-de Jong B, Kamps WA, Postma A. The willingness of general practitioners to be involved in the follow-up of adult survivors of childhood cancer. J Cancer Survivorship. 2007;1(4):292-297. doi:10.1007/s11764-0070032-z

39. Nathan PC, Daugherty CK, Wroblewski KE, et al. Family physician preferences and knowledge gaps regarding the care of adolescent and young adult survivors of childhood cancer. J Cancer Survivorship. 2013;7(3):275-282. doi:10.1007/s11764-013-0271-0

40. Sima JL, Perkins SM, Haggstrom DA. Primary care physician perceptions of adult survivors of childhood cancer. J Pediatr Hematol Oncol. 2014;36(2):118. doi:10.1097/MPH.0000000000000061

41. Oeffinger KC, Mertens AC, Hudson MM, et al. Health care of young adult survivors of childhood cancer: a report from the Childhood Cancer Survivor Study. The Ann Family Med. 2004;2(1):61-70.

42. Berg CJ, Stratton E, Esiashvili N, Mertens A. Young adult cancer survivors' experience with cancer treatment and follow-up care and perceptions of barriers to engaging in recommended care. J Cancer Educ. 2016;31(3):430-442. doi:10.1007/s13187-015-0853-9

43. Miller KA, Wojcik KY, Ramirez CN, et al. Supporting long-term follow-up of young adult survivors of childhood cancer: correlates of healthcare self-efficacy. Pediatr Blood Cancer. 2017;64(2):358-363. doi:10.1002/pbc. 26209

44. Kirchhoff AC, Montenegro RE, Warner EL, et al. Childhood cancer survivors' primary care and follow-up experiences. Supportive Care in Cancer. 2014;22(6):1629-1635. doi:10.1007/s00520-014-2130-6

45. Signorelli C, Wakefield CE, Fardell JE, et al. The role of primary care physicians in childhood cancer survivorship care: multiperspective interviews. Oncologist. 2018;23:1-10.

46. Basso G, Pillon M, Schiavo S, Varotto S, Tremolada M. Patient satisfaction in Italian childhood cancer survivors: human aspects of treatment as a key factor in patients' quality of life. Health Soc Work. 2015;40(4):e148-e155.

47. Seehusen DA, Baird D, Bode D. Primary care of adult survivors of childhood cancer. Am Fam Physician. 2010;81(10):1250-1255.

\section{Publish your work in this journal}

Cancer Management and Research is an international, peer-reviewed open access journal focusing on cancer research and the optimal use of preventative and integrated treatment interventions to achieve improved outcomes, enhanced survival and quality of life for the cancer patient.
The manuscript management system is completely online and includes a very quick and fair peer-review system, which is all easy to use. Visit http://www.dovepress.com/testimonials.php to read real quotes from published authors. 